\title{
Erratum: Flexible scheme for the implementation of nonadiabatic geometric quantum computation [Phys. Rev. A 101, 032322 (2020)]
}

\author{
Yi-Hao Kang ${ }^{\circ}$, Zhi-Cheng Shi, Bi-Hua Huang, Jie Song, and Yan Xia
}

(Received 4 April 2020; published 23 April 2020)

DOI: 10.1103/PhysRevA.101.049902

In Appendix D, there exist errors in the derivations of Eqs. (D9) and (D10) for the estimation of the rank of matrix $\mathcal{M}(t)$. Here, we would like to show the result that the matrix $\mathcal{M}(t)$ is still not full rank but the estimation of rank of $\mathcal{M}(t)$ is different from that given by Eq. (D10). The result only corrects the estimation of the rank of matrix $\mathcal{M}(t)$ given in Eq. (D10) and does not influence other results of the original paper.

In fact, for the dynamic invariant,

$$
I(t)=\sum_{\wp<\wp^{\prime}} \tilde{\xi}_{\wp, \wp}(t) \tilde{G}_{\wp, \wp^{\prime}},
$$

with $\tilde{G}_{\wp, \wp} \in \operatorname{so}(N)$, noting that $I(t)$ is a Hermitian and antisymmetric matrix, the nonzero eigenvalues of $I(t)$ are always pairs of positive and negative real numbers. Therefore, we can rewrite $I(t)$ as

$$
I(t)=\sum_{n} \epsilon_{n}\left[\left|\phi_{n}^{+}(t)\right\rangle\left\langle\phi_{n}^{+}(t)|-| \phi_{n}^{-}(t)\right\rangle\left\langle\phi_{n}^{-}(t)\right|\right] .
$$

Here, $\left|\phi_{n}^{ \pm}(t)\right\rangle$ is the eigenvector of $I(t)$ with nonzero eigenvalue $\pm \epsilon_{n}$, satisfying $\left[\left|\phi_{n}^{ \pm}(t)\right\rangle\right]^{\mathrm{T}}=\left\langle\phi_{n}^{\mp}(t)\right|$ because of $I^{\mathrm{T}}(t)=-I(t)$. We assume that the rank of $I(t)$ is $2 \mu$ with $\mu$ as a positive integer and the dimension of the $\operatorname{kernel} \mathcal{K}=\operatorname{ker}[I(t)]$ of $I(t)$ is $N-2 \mu$. When $\mu \geqslant 2$, we can easily find a Hermitian and antisymmetric matrix as

$$
X(t)=\epsilon_{n^{\prime}}\left[\left|\phi_{n}^{+}(t)\right\rangle\left\langle\phi_{n}^{+}(t)|-| \phi_{n}^{-}(t)\right\rangle\left\langle\phi_{n}^{-}(t)\right|\right]-\epsilon_{n}\left[\left|\phi_{n^{\prime}}^{+}(t)\right\rangle\left\langle\phi_{n^{\prime}}^{+}(t)|-| \phi_{n^{\prime}}^{-}(t)\right\rangle\left\langle\phi_{n^{\prime}}^{-}(t)\right|\right],
$$

with $n \neq n^{\prime}$, which satisfies $I(t) X(t)=0$. Since any Hermitian and antisymmetric matrix can be spanned by the generators of $\operatorname{so}(N)$, we have $X(t) \in \operatorname{so}(N), \mathcal{M}(t) X(t)=-i[I(t), X(t)]=0$. For the case of $\mu=1$, we have $N-2 \mu \geqslant 2$ when $N \geqslant 4$, and we can derive the projection operator $P_{\mathcal{K}}$ from subspace $\mathcal{K}$ as

$$
P_{\mathcal{K}}=\mathbb{1}_{N}-P_{I}, \quad P_{I}=\sum_{n}\left[\left|\phi_{n}^{+}(t)\right\rangle\left\langle\phi_{n}^{+}(t)|+| \phi_{n}^{-}(t)\right\rangle\left\langle\phi_{n}^{-}(t)\right|\right],
$$

which is a real symmetric matrix since $P_{I}^{\mathrm{T}}=P_{I}=P_{I}^{\dagger}$. Therefore, $P_{\mathcal{K}}$ has eigenvectors $\left\{\left|\phi_{0}^{n}(t)\right\rangle\right\}$ with all real components. We select two eigenvectors $\left\{\left|\phi_{0}^{1}(t)\right\rangle,\left|\phi_{0}^{2}(t)\right\rangle\right\}$ and construct a matrix $X(t)$ as

$$
X(t)=\left|\phi_{0}^{+}(t)\right\rangle\left\langle\phi_{0}^{+}(t)|-| \phi_{0}^{-}(t)\right\rangle\left\langle\phi_{0}^{-}(t)\right|,
$$

with $\left|\phi_{0}^{ \pm}(t)\right\rangle=\left[\left|\phi_{0}^{1}(t)\right\rangle \pm i\left|\phi_{0}^{2}(t)\right\rangle\right] / \sqrt{2} . X(t)$ is a Hermitian and antisymmetric matrix, thus, $X(t) \in \operatorname{so}(N)$. Moreover, we have $I(t) X(t)=0$ due to $X(t) \in \mathcal{K}$. Consequently, $\mathcal{M}(t) X(t)=-i[I(t), X(t)]=0$ can be also obtained. Therefore, when $N \geqslant 4$, we can always find two nonzero operators $I(t)$ and $X(t)$ satisfying $\mathcal{M}(t) I(t)=\mathcal{M}(t) X(t)=0$, and the rank of $\mathcal{M}(t)$ should be less or equal to $[N(N-1)] / 2-2$. Noting that $\mathcal{M}(t)$ is also an antisymmetric matrix, its rank should be even. When $[N(N-1)] / 2-2$ is odd, we have $\operatorname{rank}[\mathcal{M}(t)] \leqslant[N(N-1)] / 2-3$. For example, for $I(t) \in \operatorname{so}(4), \operatorname{rank}[\mathcal{M}(t)] \leqslant 4$, for $I(t) \in$ $\operatorname{so}(5), \operatorname{rank}[\mathcal{M}(t)] \leqslant 8$, and for $I(t) \in \operatorname{so}(6), \operatorname{rank}[\mathcal{M}(t)] \leqslant 12$. The results given above all accord with the numerical results. For $N=3$, we have $\mu=1$ and $N-2 \mu=1$; only $\mathcal{M}(t) I(t)=0$ can be found, accordingly, $\operatorname{rank}[\mathcal{M}(t)] \leqslant(3 \times 2) / 2-1=2$. 